\title{
THE VALIDITY OF HEMATOLOGIC MARKERS FOR DIAGNOSIS OF NEONATAL SEPSIS
}

\author{
Aqeela Ayub, ${ }^{1}$ Akmal Laeeq Chishti, ${ }^{2}$ Khwaja Amjad Hassen ${ }^{3}$
}

\begin{abstract}
Objective: To determine the validity of haematologic markers for sepsis screen (absolute neutrophil count (ANC), immature/total leukocytes ratio (I:T), platelets count (PC), C-reactive protein (CRP) and serum ferritin), both individually and in combination for early diagnosis of neonatal sepsis.

Methodology: This cross - sectional analytical study was conducted in Neonatal Section of Paediatric Medicine Unit II, Mayo Hospital Lahore for one year. One hundred neonates presenting with clinical sepsis were included through non-probability, purposive sampling after written informed consent. Blood sample was collected for full hematologic screening as mentioned above along with blood cultures. Data was entered and analyzed using SPSS Version 17.
\end{abstract}

Results: There were 68 male and 32 females including 31 preterm and 69 term neonates. 45 neonates were $<1$ day age, 40 neonates $1-10$ days age, 15 neonates

Ayub A. ${ }^{1}$

Department of Paeds Medicine

KEMU / Mayo Hospital, Lahore

Chishti A.L. ${ }^{2}$

Chairman Department of Paeds Medicine

KEMU / Mayo Hospital, Lahore

Hassen K.A. ${ }^{3}$

Assistant Professor Department of Paeds Medicine

KEMU / Mayo Hospital, Lahore
11 - 30 days age. Mean weight of study cases was $2.35 \pm 0.69 \mathrm{Kg}$. Sensitivity, specificity and diagnostic accuracy of ANC were $77.3 \%, 100 \%$ and $90 \%$, for $\mathrm{I} / \mathrm{T}$ ratio were $81.8 \%, 81.4 \%$ and $81 \%$, for CRP were $75 \%$, $83.9 \%$ and $80 \%$, for platelet count were $84.1 \%, 71.4 \%$ and $77 \%$, for serum ferritin were $88.6 \%, 69.6 \%$ and $78 \%$ respectively. Sensitivity, specificity and diagnostic accuracy of combination of SF + I:T was $81.8 \%, 82.1 \%$ and $82 \%$, for combination of SF + ANC + I:T were $93.2 \%, 71.4 \%$ and $81 \%$ for combination of $\mathrm{SF}+\mathrm{CRP}+\mathrm{I}: \mathrm{T}$ were $93.2 \%, 67.9 \%$ and $79 \%$, for combination of SF + I:T + PC were $90.9 \%, 58.9 \%$ and $73 \%$, for combination of SF + CRP + ANC were $95.5 \%, 69.6 \%$ and $81 \%$. Sensitivity, specificity and diagnostic accuracy of combination of all markers were $90.9 \%, 76.8 \%$ and $83 \%$ respectively.

Conclusion: Results of our study showed that we can safely rely on hematologic markers for confirmation of neonatal sepsis both singly and in combination. We suggest that these tests may help to diagnose neonatal sepsis earlier.

Key Words: Neonatal Sepsis, Diagnostic accuracy, Lethargy, Prolong rupture of membrane, Temperature instability, Hematologic markers.

\section{Introduction}

Neonatal Sepsis is a clinical syndrome characterized by signs and symptoms of infection with or without accompanying bacteraemia in the first month of life. ${ }^{1}$ The incidence of neonatal sepsis in India has been 
estimated as 30 per 1000 live - births. ${ }^{2}$ The reported incidence of neonatal sepsis varies from $7.1-38$ per 1000 live births in Asia and from 3.5 - 8.9 per 1000 live births in South America and Caribbean. ${ }^{3,4}$ The estimated mortality rate is as high as $30 \%$ and in some studies up to $69 \% .^{5}$ WHO estimates show that one million deaths occur every year due to the neonatal sepsis ( $10 \%$ of $<5$ year mortality). Forty two percent of these deaths occur during $1^{\text {st }}$ week of life. ${ }^{6,7}$ The most important risk factors are prolonged rupture of membrane (PROM), maternal pyrexia, prematurity, low birth weight and prolonged hospitalization in the neonatal intensive care units. ${ }^{3,8,9}$

Early warning signs and symptoms are often nonspecific and can easily be confused with non-infective causes. The common clinical features of neonatal sepsis are lethargy, poor feeding, temperature instability and skin mottling. However, some of the neonates may manifest with life threatening involvement of cardiac and respiratory systems. ${ }^{10,11}$

Positive blood culture is considered gold standard for diagnosis of micro-biological infection. However, blood cultures are positive only in $5-10 \%$ of suspected sepsis cases, even at highly resourced facilities because of earlier exposure to antibiotics before samples are withdrawn. Moreover, biological culture results are usually unavailable before $48-72$ hours. Hence a reliable infection marker or a set of markers are needed to promptly and accurately identify the infected cases. $^{11,12}$

The simple hematologic screen (absolute neutronphil count, immature/total leukocyte ratio, platelet count, C-reactive protein and serum ferritin) are useful markers for the early diagnosis of neonatal sepsis. Local data suggests that CRP was positive in $80.5 \%$ of probable sepsis with a specificity of $95 \%$. Another Egyptian study showed CRP as best laboratory test with an overall sensitivity and specificity of $86 \%$ and $97 \%$ respectively. ${ }^{10,13,14}$

Total and differential leukocyte counts did not show promising results in a study but thrombocytopenia was present in $50 \%$ cases of culture positive sepsis. Another study showed the sensitivity of platelet count in proven sepsis group was $64.3 \%$. However, thrombocytopenia is a non specific indicator of infection. Absolute neutrophil count was the second most sensitive test having sensitivity of $71.4 \%$ for proven and $63.9 \%$ of probable sepsis and had a specificity of $88 \% .{ }^{12}$ Likewise in another study, ANC had sensitivity as low as $13 \%$. $^{3,13-15}$

Serum ferritin is considered as positive acute pha- se reactant. One study concluded that ferritin was raised in children with septic shock. ${ }^{16}$ While in another study it was shown that serum ferritin levels were higher in two groups with sepsis, but compared with the healthy group, the difference was statistically insignificant in newborns. ${ }^{17}$ Extensive work has been done in developed countries but limited data is available on this controversial subject in developing countries. Thus, the current study is designed to analyze various parameters of sepsis screening singly and in combination, to help formulate a guide - line for the diagnosis of neonatal sepsis. The objective of this study was to determine the validity of hematologic markers for sepsis screen (absolute neutrophil count, immature / total leukocytes ratio, platelets count, Creactive protein and serum ferritin) both individually and in combination for early diagnosis of neonatal sepsis.

\section{Operational Definitions}

Clinical Sepsis: According to IMNCI, sepsis was defined as presence of any sign listed implying high suspicion of serious bacterial infection: (convulsions, respiratory rate $>60$ breaths $/ \mathrm{min}$, severe chest indrawings, temperature $>37.7^{\circ} \mathrm{C}$ or $<35.5^{\circ} \mathrm{C}$, lethargic or unconsciousness, not able to feed/sucking, bulging fontanel, pus draining from the ear, grunting /nasal flaring, redness around umbilicus). Proven sepsis was defined as patients with positive blood cultures were categorized as having proven sepsis. Patients with negative blood cultures but abnormal hematologic results suggestive of sepsis were categorized as probable sepsis.

Hematologic Indicators of Sepsis: They were identified as absolute neutrophil count $<500 /$ cumm, imamture / total leukocyte ratio > 1:5, platelet count < $150,000 /$ cumm, CRP $>$ or $=5 \mathrm{mg} /$ litre, serum Ferritin $>140 \mathrm{ng} / \mathrm{ml}$.

\section{Material and Methods}

This cross - sectional analytical study was carried out in Neonatal Section of Paediatric Medicine Unit II, Mayo Hospital, Lahore over one year (Jan - Dec 2013). One hundred neonates presenting with clinical sepsis were included with non-probability, purposive sampling. The sample size was calculated using WHO 
software by taking incidence $38 / 1000$ with $5 \%$ level of significance and $3.8 \%$ margin of error. Inclusion Criteria were neonates (age $1^{\text {st }}-28$ days of life) of either sex, having any of the IMNCI signs of clinical sepsis. Neonates weighing $<1000$ gm, gestational age $<28$ weeks, H/O prior use of antibiotics and major systemic congenital anomalies were excluded. One hundred neonates of both genders with clinical signs of sepsis presenting in Neonatal Section at Department of Paediatrics Unit II, Mayo Hospital, Lahore, were enrolled for the study after obtaining informed written consent from the parents. Information was collected on structured questionnaires that included age, sex, anthropometric data and systemic details. A full hematologic screening including absolute neutrophil count, imamture / total leukocyte ratio, platelet count, CRP and serum ferritin along with blood cultures were obtained before the start of anti-microbial therapy. Samples were collected for blood complete picture including ANC, I/T ratio, platelet count and serum ferritin levels. Sample was collected for estimation of CRP by latex agglutination technique (quantitative). Under strict aseptic measures, $1.5 \mathrm{ml}$ venous blood was collected in blood culture bottles. The laboratory investigations were done at Paediatric Pathology Laboratory under supervision of qualified hematologist and bio-chemist. PC was done in the CBC using Sysmax method in heparinized / oxalate containing blood. ANC was calculated using formula of TLC $\mathrm{x}$ Neutrophil count / 100. I:T ratio was done by direct blood smears, stained with Giemsa and band neutrophild were counted. SF

Table 1: Baseline Characteristics of Study Newborns ( $\mathrm{n}=$ 100).

\begin{tabular}{|l|l|l|}
\hline \multicolumn{1}{|c|}{ Characteristic } & & \multicolumn{1}{c|}{ Number } \\
\hline \multirow{2}{*}{ Sex } & Male & 68 \\
\cline { 2 - 3 } & Female & 32 \\
\hline \multirow{4}{*}{ Age at Admission } & $0-1$ Day & 45 \\
\cline { 2 - 3 } & $1-10$ days & 40 \\
\cline { 2 - 3 } & $11-28$ days & 15 \\
\hline \multirow{2}{*}{ Gestational Age } & Preterm & 31 \\
\cline { 2 - 3 } & Term & 69 \\
\hline \multirow{2}{*}{$\begin{array}{l}\text { Mean Weight at } \\
\text { Admission }\end{array}$} & Preterm & $1.74 \mathrm{Kg}+/-0.56$ \\
\cline { 2 - 3 } & Term & $2.63 \mathrm{Kg}+/-0.55$ \\
\hline
\end{tabular}

was done by ELISA method. CRP was done by using rapid slide agglutination test for qualitative and semi quantitative detection. Data was entered and analyzed using SPSS Version 17. For analysis of the results, the neonates were stratified into two groups, the confirmed cases of neonatal sepsis with blood culture as positive cases (gold standard) and symptomatic neonates having clinical diagnosis of sepsis but negative with blood culture were labelled as negative cases. Then all positive and negative cases were verified through all 5 tests (ANC, IT ratio, platelet count, CRP and serum ferritin). After verification, cases were labelled true positive, false positive, false negative and true negative. Then $2 \times 2$ tables were generated to calculate specificity, sensitivity and diagnostic accuracy of hematologic markers.

\section{Results}

A total 100 neonates with clinical signs of sepsis were studied. Their sex, age, weight at admission and gestational age distribution is shown in Table $1.85 \%$ neonates presented with early onset while $15 \%$ presented with late onset neonatal sepsis. There were $68 \%$ male and $32 \%$ female cases $(2: 1)$ showing increased male predisposition. There were $31 \%$ preterm and $69 \%$ term babies. The mean weight of study cases was $2.35 \pm$ $0.69 \mathrm{Kg}$. The mean weight of pre-term neonates $(1.74$ $\pm 0.56 \mathrm{Kg}$ ) was significantly less than mean weight of term neonates $(2.63 \pm 0.55 \mathrm{Kg})$. The blood markers were studied and compared to blood culture as gold standard and results shown with statistical significance as shown in Tables $(3-12)$.

Table 2: Clinical Features (IMNCI) at admission in Study Cases $(n=100)$.

\begin{tabular}{|l|c|}
\hline \multicolumn{1}{|c|}{ Clinical Feature } & Frequency \\
\hline Respiratory symptoms & $88 \%$ \\
\hline Poor Feeding / Refusal to feed & $83 \%$ \\
\hline Tachypnea $>60 / \mathrm{min}$ & $63 \%$ \\
\hline Grunting & $5 \%$ \\
\hline Apnea & $4 \%$ \\
\hline Convulsions & $4 \%$ \\
\hline Umbilical redness / discharge & 0 \\
\hline
\end{tabular}


Table 3: Validity of Absolute Neutrophil Count Taking Blood Culture as Gold Standard.

\begin{tabular}{|c|c|c|c|c|}
\hline \multicolumn{2}{|c|}{} & \multicolumn{2}{|c|}{ Blood Culture } & \multirow{2}{*}{ Total } \\
\cline { 3 - 5 } \multicolumn{2}{|c|}{} & Positive & Negative & \\
\hline \multirow{3}{*}{ ANC } & $<500 /$ cumm & 34 & 0 & 34 \\
\cline { 2 - 5 } & $>500 /$ cumm & 10 & 56 & 66 \\
\hline \multicolumn{2}{|c|}{ Total } & 44 & 56 & 100 \\
\hline
\end{tabular}

Sensitivity $=77.3 \%$, Specificity $=100 \%, \mathrm{PPV}=100 \%$, NPV $=84.8 \%$, Diagnostic accuracy $=90 \%$

Table 4: Validity of Immature/ Total Leukocyte Ratio Taking Blood Culture as Gold Standard.

\begin{tabular}{|c|c|c|c|c|}
\hline \multicolumn{2}{|c|}{} & \multicolumn{2}{c|}{ Blood Culture } & \multirow{2}{*}{ Total } \\
\cline { 3 - 4 } \multicolumn{2}{|c|}{} & Positive & Negative & \\
\hline \multirow{2}{*}{$\begin{array}{c}\text { I/T } \\
\text { Ratio }\end{array}$} & $>1: 5$ & 36 & 11 & 47 \\
\cline { 2 - 4 } & $<1: 5$ & 8 & 45 & 53 \\
\hline \multicolumn{2}{|c|}{ Total } & 44 & 56 & 100 \\
\hline
\end{tabular}

Sensitivity $=81.8 \%$, Specificity $=81.4 \%, \mathrm{PPV}=76.6 \%$, $\mathrm{NPV}=84.9 \%$. Diagnostic accuracy $=81 \%$

Table 5: Validity of C-Reactive Protein Taking Blood Culture as Gold Standard.

\begin{tabular}{|c|c|c|c|c|}
\hline \multicolumn{2}{|c|}{} & \multicolumn{2}{c|}{ Blood Culture } & \multirow{2}{*}{ Total } \\
\cline { 3 - 5 } \multicolumn{2}{|c|}{} & Positive & Negative & \\
\hline \multirow{2}{*}{ CRP } & $>5 \mathrm{mg} / \mathrm{l}$ & 33 & 9 & 42 \\
\cline { 2 - 4 } & $<5 \mathrm{mg} / \mathrm{l}$ & 11 & 47 & 58 \\
\hline \multicolumn{2}{|c|}{ Total } & 44 & 56 & 100 \\
\hline
\end{tabular}

Sensitivity $=75 \%$, Specificity $=83.9 \%, \mathrm{PPV}=78.6 \%$,

NPV $=81 \%$, Diagnostic accuracy $=80 \%$

Table 6: Validity of Platelet Count Taking Blood Culture as Gold Standard.

\begin{tabular}{|l|c|c|c|c|}
\hline \multicolumn{2}{|c|}{} & \multicolumn{2}{c|}{ Blood Culture } & \multirow{2}{*}{ Total } \\
\cline { 3 - 5 } \multicolumn{2}{|c|}{} & Positive & Negative & \\
\hline \multirow{2}{*}{$\begin{array}{l}\text { Platelet } \\
\text { Count }\end{array}$} & $<150,000$ & 37 & 16 & 53 \\
\cline { 2 - 4 } & $>150,000$ & 7 & 40 & 47 \\
\hline \multicolumn{2}{|c|}{ Total } & 44 & 56 & 100 \\
\hline
\end{tabular}

Sensitivity $=84.1 \%$, Specificity $=71.4 \%, \mathrm{PPV}=69.8 \%$, $\mathrm{NPV}=85.1 \%$, Diagnostic accuracy $=77 \%$
Table 7: Validity of Serum Ferritin Taking Blood Culture as Gold Standard.

\begin{tabular}{|l|c|c|c|c|}
\hline \multicolumn{2}{|c|}{} & \multicolumn{2}{c|}{ Blood Culture } & \multirow{2}{*}{ Total } \\
\cline { 3 - 5 } \multicolumn{2}{|c|}{} & Positive & Negative & \\
\hline \multirow{2}{*}{$\begin{array}{l}\text { Serum } \\
\text { Ferritin }\end{array}$} & $>120 /$ cumm & 39 & 17 & 56 \\
\cline { 2 - 5 } & $<120 /$ cumm & 5 & 39 & 44 \\
\hline \multicolumn{2}{|c|}{ Total } & 44 & 56 & 100 \\
\hline
\end{tabular}

Sensitivity $=88.6 \%$, Specificity $=69.6 \%, \mathrm{PPV}=69.6 \%$, $\mathrm{NPV}=88.6 \%$, Diagnostic accuracy $=78 \%$

Table 8: Validity of SF+I:T Taking Blood Culture as Gold Standard.

\begin{tabular}{|c|l|c|c|c|}
\hline \multicolumn{2}{|c|}{} & \multicolumn{2}{c|}{ Blood Culture } & \multirow{2}{*}{ Total } \\
\cline { 3 - 5 } \multicolumn{2}{|c|}{ SF+I:T } & Positive & Negative & \\
\hline \multirow{3}{*}{} & Positive & 36 & 10 & 46 \\
\cline { 2 - 5 } & Negative & 8 & 46 & 54 \\
\hline \multicolumn{2}{|c|}{ Total } & 44 & 56 & 100 \\
\hline
\end{tabular}

Sensitivity $=81.8 \%$, Specificity $=82.1 \%, \mathrm{PPV}=78.26 \%$, $\mathrm{NPV}=85.19 \%$, Diagnostic accuracy $=82.0 \%$

Table 9: Validity of SF+ANC+IT Taking Blood Culture as Gold Standard.

\begin{tabular}{|c|l|c|c|c|}
\hline \multicolumn{2}{|c|}{} & \multicolumn{2}{c|}{ Blood Culture } & \multirow{2}{*}{ Total } \\
\cline { 3 - 5 } \multicolumn{2}{|c|}{} & Positive & Negative & \\
\hline \multirow{3}{*}{ SF+ANC+IT } & Positive & 41 & 16 & 57 \\
\cline { 2 - 5 } & Negative & 3 & 40 & 43 \\
\hline \multicolumn{2}{|c|}{ Total } & 44 & 56 & 100 \\
\hline
\end{tabular}

Sensitivity $=93.2 \%$, Specificity $=71.4 \%, \mathrm{PPV}=71.9 \%$, $\mathrm{NPV}=93.0 \%, \mathrm{DA}=81.0 \%$

Table10: Validity of SF+CRP+I:T taking Blood Culture as Gold Standard.

\begin{tabular}{|c|l|c|c|c|}
\hline \multicolumn{2}{|c|}{} & \multicolumn{2}{c|}{ Blood Culture } & \multirow{2}{*}{ Total } \\
\cline { 3 - 5 } \multicolumn{2}{|c|}{} & Positive & Negative & \\
\hline \multirow{2}{*}{ SF+CRP+IT } & Positive & 41 & 18 & 59 \\
\cline { 2 - 5 } & Negative & 3 & 38 & 41 \\
\hline \multicolumn{2}{|c|}{ Total } & 44 & 56 & 100 \\
\hline
\end{tabular}

Sensitivity $=93.2 \%$, Specificity $=67.9 \%, \mathrm{PPV}=64.5 \%$, $\mathrm{NPV}=92.7 \%, \mathrm{DA}=79.0 \%$ 
Table 11: Validity of SF+IT+PC Taking Blood Culture as Gold Standard.

\begin{tabular}{|c|l|c|c|c|}
\hline \multicolumn{2}{|c|}{} & \multicolumn{2}{c|}{ Blood Culture } & \multirow{2}{*}{ Total } \\
\cline { 3 - 5 } \multicolumn{2}{|c|}{} & Positive & Negative & \\
\hline \multirow{3}{*}{ SF+IT+PC } & Positive & 40 & 23 & 63 \\
\cline { 2 - 5 } & Negative & 4 & 33 & 37 \\
\hline \multicolumn{2}{|c|}{ Total } & 44 & 56 & 100 \\
\hline
\end{tabular}

Sensitivity $=90.9 \%$, Specificity $=58.9 \%, \mathrm{PPV}=63.5 \%$, $\mathrm{NPV}=89.2 \%, \mathrm{DA}=73.0 \%$

Table 12: Validity of SF+CRP+ANC Taking Blood Culture as Gold Standard.

\begin{tabular}{|c|l|c|c|c|}
\hline \multicolumn{2}{|c|}{} & \multicolumn{2}{c|}{ Blood Culture } & \multirow{2}{*}{ Total } \\
\cline { 3 - 5 } \multicolumn{2}{|c|}{} & Positive & Negative & \\
\hline \multirow{3}{*}{ SF+CRP+ANC } & Positive & 42 & 17 & 59 \\
\cline { 2 - 5 } & Negative & 2 & 39 & 41 \\
\hline \multicolumn{2}{|c|}{ Total } & 44 & 56 & 100 \\
\hline
\end{tabular}

Sensitivity $=95.5 \%$, Specificity $=69.6 \%, \mathrm{PPV}=71.2 \%$, $\mathrm{NPV}=69.6 \%, \mathrm{DA}=81.0 \%$

Table 13: Validity of Combination of All Tests Taking Blood Culture as Gold Standard.

\begin{tabular}{|l|l|c|c|c|}
\hline \multicolumn{2}{|c|}{} & \multicolumn{2}{c|}{ Blood Culture } & \multirow{2}{*}{ Total } \\
\cline { 3 - 4 } \multicolumn{2}{|c|}{} & Positive & Negative & \\
\hline \multirow{2}{*}{$\begin{array}{l}\text { All tests } \\
\text { combined }\end{array}$} & Positive & 40 & 13 & 53 \\
\cline { 2 - 5 } & Negative & 4 & 43 & 47 \\
\hline \multicolumn{2}{|c|}{ Total } & 44 & 56 & 100 \\
\hline
\end{tabular}

Sensitivity $=90.9 \%$, Specificity $=76.8 \%, \mathrm{PPV}=75.5 \%$, $\mathrm{NPV}=91.5 \%$, Diagnostic accuracy $=83 \%$

\section{Discussion}

Early diagnosis of neonatal septicemia is difficult. ${ }^{10}$ The positive blood culture report which is a gold standard for the diagnosis of neonatal sepsis requires 48 72 hours of waiting time. Further, the yield of the blood culture is also low and in some hospitals, the facileties may not be available. Hence, for early identification, several screening tests and their usefulness, either individually or in combinations, have been reported.
Recently, few researchers suggested the need for the reassessment of the CBC. They did not use CRP as a screening tool and reported a higher sensitivity of the physical examination. ${ }^{11,12}$

Simple blood screening tests such as absolute neutrophil count (ANC), immature to total leukocyte Ratio (I: T), platelet count (PC), C - reactive protein (CRP) and serum ferritin (SF) for early detection of neonatal septicaemia were studied. The ratio of early neonatal sepsis was higher than late onset sepsis. One study also reported that frequency of early neonatal sepsis $(54.53 \%)$ was higher than late neonatal sepsis $(45.57 \%)$, without statistical significance. ${ }^{18}$ One study reported that $67 \%$ of cases developed neonatal septicemia before seven days. ${ }^{19}$ Regarding sex distribution, our results compare with another study which reported the proportion cases of neonatal sepsis was higher in male $(60 \%)$ than female neonates. ${ }^{19}$ But another study reported that risk of developing neonatal sepsis was nearly equal in both genders (57\% male and $43 \%$ female). However no statistically significant difference was found. ${ }^{18}$ Gender predisposition to sepsis remained controversial, while a study done in Winthrop University hospital showed no gender difference in the frequency of bacterial sepsis; while several other studies had shown that the frequency and even severity of bacterial sepsis was higher in males. ${ }^{20}$ One case series reported the frequency of preterm neonates developing neonatal sepsis being $88.3 \% .^{19,21}$

Mothers of $83 \%$ neonates complained about baby's poor feeding. This may contribute towards developing neonatal sepsis as mother's milk is important source of immune factors in neonates. Poor feeding or refusal to feed remains an important symptom of neonatal sepsis.

For ANC, sensitivity, specificity, PPV and NPV were $77.3 \%, 100 \%, 100 \%$ and $84.8 \%$ respectively. The overall diagnostic accuracy of ANC was calculated as $90 \%$. But one study reported sensitivity for ANC was little higher than that of our study $84 \%{ }^{22}$

For I/T ratio, sensitivity, specificity, PPV and NPV were $81.8 \%, 81.4 \%, 76.6 \%$ and $84.9 \%$ respectively. The overall diagnostic accuracy of I/T ratio was estimated as $81 \%$. These results were bit different from other studies. One study reported the sensitivity and specificity of I/T ratio was $63 \%$ and $85 \%$ respectively. ${ }^{23}$ But another study reported the sensitivity, specificity, PPV and NPV for I/T ratio were 33\%, $100 \%, 100 \%$ and $66 \%$ respectively ${ }^{(22}$ Thus conflicting results are observed between different studies and require more studies to confirm the validity of these 
markers.

For CRP as sepsis indicator, the estimated sensitivity, specificity, PPV and NPV were 75\%, $83.9 \%$, $78.6 \%$ and $81 \%$ respectively. The overall diagnostic accuracy of CRP was calculated as $80 \%$. One study reported the sensitivity and specificity of CRP was $84 \%$ and $65 \%$ respectively. $^{23}$

For Platelet Count (PC), sensitivity, specificity, PPV and NPV were 84.1\%, 71.4\%, 69.8\% and 85.1\% respectively. The overall diagnostic accuracy of PC was calculated as $77 \%$. But one study reported the sensitivity and specificity of PC $88 \%$ and $51.4 \%$ with an accuracy of $56 \% .{ }^{24}$ Another study reported the sensitivity, specificity, PPV and NPV for PC were $48 \%$, $96 \%, 91 \%$ and $71 \%$ respectively. ${ }^{22}$

We also performed estimation of serum ferritin (SF) levels and found the sensitivity, specificity, PPV and NPV to be $88.6 \%, 69.6 \%, 69.6 \%$ and $88.6 \%$ respectively. The overall diagnostic accuracy was calculated as $78 \%$. One study reported the sensitivity and specificity of SF as $100 \%$ and $58 \%$, respectively. ${ }^{16}$

Various combinations of blood markers in sepsis had been studied earlier ${ }^{15}$ to determine the diagnostic accuracy in neonatal sepsis and found valid. We found that sensitivity, specificity, PPV and NPV of combination of $\mathrm{ANC}+\mathrm{CRP}+\mathrm{I}: \mathrm{T}$ ratio were $86.4 \%, 32.1 \%$, $50 \%$ and $75 \%$ respectively. The overall diagnostic accuracy was $56 \%$. One study reported that I:T ratio+ ANC+CRP was the best combination which gave $88 \%$ sensitivity, $84 \%$ specificity, $86 \% \mathrm{PPV}$ and $85 \%$ NPV. ${ }^{19}$ We found that sensitivity, specificity, PPV and $\mathrm{NPV}$ of combination of $\mathrm{ANC}+\mathrm{CRP}+\mathrm{I}: \mathrm{T}+\mathrm{PC}+\mathrm{SF}$ were $90.9 \%, 76.8 \%, 75.5 \%$ and $91.5 \%$ respectively. The overall diagnostic accuracy was $83 \%$.

Thus our study showed higher sensitivity for SF (88\%), followed by PC $(84 \%)$, I:T ratio $(81 \%)$, ANC (77\%) and CRP (75\%). On the other hand higher specificity was shown by ANC (100\%), followed by CRP $(83 \%)$, IT ratio $(81 \%), \mathrm{CRP}(80 \%), \mathrm{SF}(78 \%)$ and $\mathrm{PC}$ $(77 \%)$.

The combination of different tests showed higher sensitivity $95.5 \%$ for a combination of $\mathrm{SF}+\mathrm{CRP}+\mathrm{ANC}$ followed by $93.2 \%$ for $(\mathrm{SF}+\mathrm{IT}+\mathrm{ANC})$ and $93.2 \%$ for SF+IT+CRP. Diagnostic accuracy on the other hand was found higher $83 \%$ with combination of all 5 tests (ANC+CRP+SF+PC+IT) and $82 \%$ with $(\mathrm{SF}+\mathrm{IT})$. This was followed by $81 \%$ both with $(\mathrm{SF}+\mathrm{CRP}+\mathrm{ANC})$ and $(\mathrm{SF}+\mathrm{IT}+\mathrm{ANC})$.

\section{Conclusion}

Results showed that hematologic markers for diagnosis of neonatal sepsis were quite reliable and valid. These blood tests can be used either singly or in combination. Both SF and I:T ratio were reliable indicators for early diagnosis of neonatal sepsis when used singly. However, SF+CRP+ANC, SF+I:T+ANC and SF+ $\mathrm{CRP}+\mathrm{I}: \mathrm{T}$ had also proven to be reliable combination for diagnosis of neonatal sepsis. Our results are promising and suggest their judicious use. However by adding results from further studies will help make uniform recommendations to develop standard protocols.

\section{References}

1. Shankar MJ, Agarwal R, Deorari AK. Sepsis in the new born. Indian Journal of Pediatrics, 2008; 75 (3): 261-70.

2. Department of Pediatrics (2002-03), National Neonatal Perinatal Database. [online] New Delhi: All India Institute of Medical Sciences; NNPD nodal center.

3. Stoll BJ. Infections of the Neonatal Infant. In: Behrman RE, Kaliegman RM, Jenson HB, Stanton BF editors. Nelson textbook of Pediatrics. 18th ed: Philadelphia: WB Saunders Company; 2008: pp. 794-811.

4. Vergnano S, Sharland M, Kazembe P, Mwansambo C, Heath PT. Neonatal sepsis: an international perspective. Arch Dis Child Fetal Neonatal Ed. 2005; 90 (3): F2204.

5. Zuppa AA, Calabrese V, D'Andrea V, Fracchiolla A, Scorrano A, Orchi C, et al. Evaluation of C-reactive protein and other immunologic markers in diagnosis of neonatal sepsis. Minerva Pediatr. 2007; 59 (3): 267-74.

6. Lawn JE, Cousens S, Zupan J. 4 million neonatal deaths: when? Where? Why? Lancet. 2005; 365 (9462): 891-900.

7. Gomella TL, Cunningham MD, Eyal FG, Tuttle D. Neonatology. 6th ed. New York: McGraw-Hill. 2009: pp. 665-72.

8. Darmstadt GL, Bhutta ZA, Cousens S, Adam T, Walker $\mathrm{N}$, de Bernis L. Evidence - based, cost - effective interventions: how many newborn babies can we save? Lancet. 2005; 365 (9463): 977-88.

9. Bahl R, Martines J, Ali N, Bhan MK, Carlo W, Chan $\mathrm{KY}$, et al. Research priorities to reduce global mortality from newborn infections by 2015. Pediatr Infect Dis J. 2009; 28 (1 Suppl): S43-8.

10. Zaki Mel S, el-Sayed H. Evaluation of microbiologic and hematologic parameters and E-selectin as early predictors for outcome of neonatal sepsis. Arch Pathol Lab Med. 2009; 133 (8): 1291-6.

11. Darmstadt GL, Batra M, Zaidi AK. Parenteral antibiotics for the treatment of serious neonatal bacterial 
infections in developing country settings. Pediatr Infect Dis J. 2009; 28 (1 Suppl): S37-42.

12. Edmond K, Zaidi A. New approaches to preventing, diagnosing, and treating neonatal sepsis. PLoS Med. 2010; 7 (3): e1000213.

13. Mannan MA, Shahidullah M, Noor MK, Islam F, Alo D, Begum NA. Utility of C-reactive protein and hematological parameters in the detection of neonatal sepsis. Mymensingh Med J. 2010; 19 (2): 259-63.

14. Waliullah SM, Islam MN, Siddika M, Hossain MA, Jahan I, Chowdhury AK. Evaluation of simple hematological screen for early diagnosis of neonatal sepsis. Mymensingh Med J. 2010; 19 (1): 41-7.

15. Ahmed Z, Ghafoor T, Waqar T, Ali S, Aziz S, Mahmud $\mathrm{S}$. Diagnostic value of $\mathrm{C}$ - reactive protein and haematological parameters in neonatal sepsis. J Coll Physicians Surg Pak. 2005; 15 (3): 152-6.

16. Garcia PC, Longhi F, Branco RG, Piva JP, Lacks D, Tasker RC. Ferritin levels in children with severe sepsis and septic shock. Acta Paediatr. 2007; 96 (12): 182931.

17. Yapakci E, Tarcan A, Celik B, Ozbek N, Gurakan B. Serum pro-hepcidin levels in term and preterm newborns with sepsis. Pediatr Int. 2009; 51 (2): 289-92.

Neurology, Neurosurgery and Psychiatry. 2002; 73 (suppl 1): i17-i22.
18. Sadiq ZM, Al-Anee AH. Sepsis in Neonatology Unit of Kirkuk Pediatric Hospital. Journal of Kirkuk University, 2010; 5 (1): 1-7.

19. Buch AC, Srivastava V, Kumar H, Jadhav PS. Evaluation of haemotological profile in early diagnosis of clinically suspected cases of neonatal sepsis. International Journal of Basic and Applied Medical Sciences, 2011; 1 (1): 1-6.

20. Danai PA, Dannino DM, Moss M, Martin GS. The epidemiology of sepsis among patients with cancer. Chest, 2006; 129: 1432-40.

21. Feigin RD, Cherry JD. Textbook of pediatric infectious diseases. Philadelphia: WB Saunders, 1998: pp. 892926.

22. Bhandari V, Wang C, Rinder C, Rinder H. Hematologic profile of sepsis in neonates: neutrophil CD64 as a diagnostic marker. Pediatrics. 2008; 121 (1): 129-34.

23. Chakraborty D, Nag D, Bandyopadhyay R, Mondal S, Sinha S. Neonatal sepsis: Role of a battery of immunohematological tests in early diagnosis. Int J App Basic Med Res. 2012; 2: 43-7.

24. Noor MK, Shahidullah M, Rahman H, Mutanabbi M. Interleukin-6: A Sensitive Parameter for the Early Detection of Neonatal Sepsis. Bangabandhu Sheikh Mujib Medical University Journal, 2009; 1 (1): 1-5. 